\title{
The Weil-étale topology on schemes over finite fields
}

\author{
S. Lichtenbaum
}

\begin{abstract}
We introduce an essentially new Grothendieck topology, the Weil-étale topology, on schemes over finite fields. The cohomology groups associated with this topology should behave better than the standard étale cohomology groups. In particular there is a very natural definition of an Euler characteristic and a plausible conjecture relating the Euler characteristic of $\mathbf{Z}$ to the value of the zeta-function at $s=0$. This conjecture is proved in certain cases.
\end{abstract}

\section{Introduction}

In this paper we introduce a new, or at least neglected, Grothendieck topology on the category of schemes of finite type over a finite field. This topology, which we call the Weil-étale topology, bears the same relation to the étale topology as the Weil group bears to the Galois group.

Recall that, if $K$ is the function field of a curve over a finite field $k, \bar{K}$ is a fixed algebraic closure of $K$, and $G_{K}$ is the Galois group of $\bar{K}$ over $K, G_{K}$ comes with a natural surjection $\pi$ to $\operatorname{Gal}(K \bar{k} / K)$, which is isomorphic to $\hat{\mathbf{Z}}$ and topologically generated by the Frobenius element $\phi$. The Weil group $W_{K}$ is just $\pi^{-1}(\mathbf{Z})$ where $\mathbf{Z}$ is the subgroup of $\operatorname{Gal}(K \bar{k} / K)$ consisting of all integral powers of $\phi$. (Of course, this works equally well if 'curve' is replaced by 'algebraic variety', but classically only curves were considered.)

The Weil-étale topology should have several advantages over the étale topology. Conjecturally, all the motivic cohomology groups in this topology should be finitely generated, whereas in the étale topology these groups can be quite complicated. Also, the Weil-étale cohomology groups should contain more information, in the sense that they should determine the étale cohomology groups, but not vice versa.

Finally, there should be a natural notion of Euler characteristic for these groups, which is closely related to special values of zeta-functions. In short, it is our hope that the Weil-étale motivic cohomology groups of algebraic varieties over finite fields should be better suited than the étale groups to any possible arithmetic application.

In the body of this paper we first prove a global duality theorem for cohomology of Weil-étale sheaves on curves over finite fields, starting from a similar theorem in the étale case, which is essentially a version of global class field theory. Our duality theorem is a Verdier-type duality and is properly stated (Theorem 5.4) in terms of the derived category of abelian groups.

We then go on to state a conjecture (Conjecture 7.3) relating the behavior of zeta-functions of algebraic varieties $V$ over finite fields at the point $s=0$ to Weil-étale Euler characteristics and prove this conjecture for curves, smooth surfaces, and arbitrary projective smooth varieties.

Received 7 April 2003, accepted in final form 14 April 2004, published online 21 April 2005.

2000 Mathematics Subject Classification 14F20 (primary), 14G10 (secondary).

Keywords: étale cohomology, zeta-functions.

The author has been partially supported by the Institut des Hautes Études Scientifiques, the Newton Institute, and NSF grant DMS-9970333.

This journal is (C) Foundation Compositio Mathematica 2005. 


\section{S. LichtenBAUM}

In the case when $V$ is projective and smooth, this is a variant of Theorem 0.4 a of [Mil86]; but if $V$ is not projective, we do not know even a conjectural statement in terms of étale cohomology.

In an earlier version of this paper, I had stated Conjecture 7.3 for arbitrary quasi-projective varieties, but it seems now as a result of recent unpublished work of Geisser that it is false in general for singular varieties of dimension 2 or more. Geisser has introduced a variant of the Weilétale topology called the Weil-eh topology, and Conjecture 7.3 should probably be stated in terms of the cohomology groups associated with this topology. This cohomology agrees with Weil-étale cohomology for smooth varieties and for curves, whence the current version of Conjecture 7.3.

It is tempting to think that Conjecture 7.3 follows from resolution of singularities, but I do not see how to show this. The problem is that, in the terminology of $\S 7$, we cannot conclude that, if two members of a short exact sequence are $\theta$-finite, then the third one is.

\section{The cohomology of $\Gamma_{0}$-modules and $\Gamma$-modules}

In order to avoid confusion, we let $\Gamma_{0}$ denote $\mathbf{Z}$ as abelian group, and $\Gamma$ denote $\hat{\mathbf{Z}}$ as topological abelian group. If $M$ is a discrete topological $\Gamma$-module, then $H^{i}(\Gamma, M)$ will denote the profinite group cohomology of $\Gamma$ with coefficients in $M$.

Lemma 1.1. Let $M$ be a discrete torsion topological $\Gamma$-module. The natural map from $H^{i}(\Gamma, M)$ to $H^{i}\left(\Gamma_{0}, M\right)$ is an isomorphism.

Proof. It is well known that $\operatorname{scd}(\Gamma)=2$, and $\operatorname{cd}(\Gamma)=\operatorname{scd}\left(\Gamma_{0}\right)=1$. So both cohomology groups are zero for $i \geqslant 2$ and the lemma is clear for $i=0$. So we may assume $i=1$. Let $\sigma$ be a generator of $\Gamma_{0}$. Then $H^{1}\left(\Gamma_{0}, M\right)$ may be identified with $M /(\sigma-1) M$, while $H^{1}(\Gamma, M)$ may be identified with $\underset{\operatorname{Lim}}{\longrightarrow}\left(M_{(n)}^{\sigma^{n}} /(\sigma-1) M^{\sigma^{n}}\right)$, where $M_{(n)}$ is the kernel of multiplication by $N_{n}=1+\sigma+\cdots+\sigma^{n-1}$ on $M$. Since $M$ is torsion, it is easy to see that $M=\bigcup_{n} M_{(n)}^{\sigma^{n}}$. (Let $x$ be in $M$. Since $\Gamma$ acts continuously on $M$, there exists an $r$ such that $\sigma^{r} x=x$ and there exists an $m$ such that $m x=0$. Then $N_{m r}(x)=0$.)

Lemma 1.2. Let $M$ be any discrete topological $\Gamma$-module. Then there are functorial isomorphisms:

(a) $H^{0}(\Gamma, M) \cong H^{0}\left(\Gamma_{0}, M\right)$;

(b) $H^{1}(\Gamma, M) \cong H^{1}\left(\Gamma_{0}, M\right)_{\text {tor }}$;

(c) $H^{2}(\Gamma, M) \cong H^{1}\left(\Gamma_{0}, M\right) \otimes_{\mathbf{Z}} \mathbf{Q} / \mathbf{Z}$.

Proof. Part (a) is clear.

To prove (b) and (c), we first assume that $M$ is torsion-free. Look at the commutative diagram

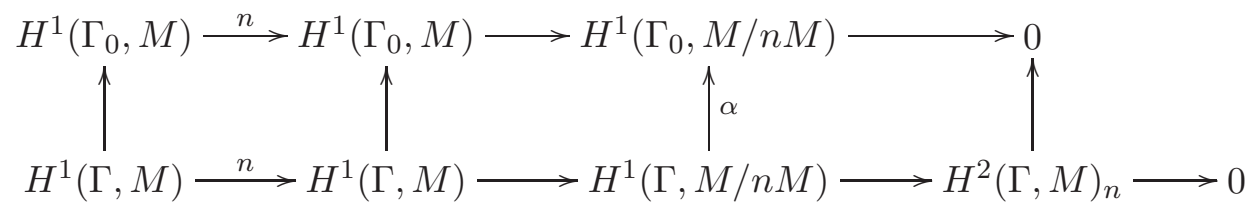

where $\alpha$ is an isomorphism by Lemma 1.1. It follows from diagram chasing that there is a natural isomorphism

$$
H^{2}(\Gamma, M)_{n} \stackrel{\sim}{\rightarrow}\left(H^{1}\left(\Gamma_{0}, M\right) \otimes(\mathbf{Z} / n \mathbf{Z})\right) /\left(H^{1}(\Gamma, M) \otimes \mathbf{Z} / n \mathbf{Z}\right)
$$

and hence in the limit an isomorphism

$$
H^{2}(\Gamma, M) \stackrel{\sim}{\rightarrow} H^{1}\left(\Gamma_{0}, M\right) \otimes \mathbf{Q} / \mathbf{Z},
$$

since $H^{2}(\Gamma, M)$ is torsion and $H^{1}(\Gamma, M) \otimes \mathbf{Q} / \mathbf{Z}=0$. 
The WeIL-ÉtAle TOPOLOGY ON SCHEMES OVER FINITE FIELDS

Since $H^{0}\left(\Gamma_{0}, M\right) \stackrel{\sim}{\rightarrow} H^{0}(\Gamma, M)$ and $H^{0}\left(\Gamma_{0}, M / n M\right) \stackrel{\sim}{\rightarrow} H^{0}(\Gamma, M / n M)$, it is immediate that $H^{1}\left(\Gamma_{0}, M\right)_{n} \stackrel{\sim}{\rightarrow} H^{1}(\Gamma, M)_{n}$, from which (b) follows.

The case of general $M$ follows easily from consideration of the exact sequence

$$
0 \rightarrow M_{\text {tor }} \rightarrow M \rightarrow M / M_{\text {tor }} \rightarrow 0 .
$$

Definition 1.3. A finitely generated $\Gamma_{0}$-module $M$ is semi-simple at zero if the natural map from $M^{\Gamma_{0}}$ to $M_{\Gamma_{0}}$ is an isomorphism modulo torsion.

Lemma 1.4. Let $M$ be a discrete finitely generated $\Gamma_{0}$-module such that the action of $\Gamma_{0}$ on $M$ can be extended to a continuous action of $\Gamma$. Then $M$ is semi-simple at zero.

Proof. Let $N=M^{\Gamma_{0}}=M^{\Gamma}$. Since $H^{1}(\Gamma, N)$ is torsion, we have an exact sequence modulo torsion:

$$
0 \rightarrow N^{\Gamma_{0}} \rightarrow M^{\Gamma_{0}} \rightarrow(M / N)^{\Gamma_{0}} \rightarrow 0
$$

It immediately follows first that both $(M / N)^{\Gamma_{0}}$ and $(M / N)_{\Gamma_{0}}$ are torsion, and then, since $N$ is obviously semi-simple at zero, so is $M$.

\section{Definition of the Weil-étale topology}

Let $k$ be a finite field and $\bar{k}$ a fixed algebraic closure of $k$. Let $X$ be a scheme of finite type over $k$ and let $\bar{X}=X \times_{k} \bar{k}$. We define the Weil-étale topology as the following Grothendieck topology: $\mathcal{W}=\mathcal{W}_{X}$ on $X$ by letting $\operatorname{Cat}(\mathcal{W})$ be the category defined as follows: the objects of Cat $(\mathcal{W})$ are étale schemes and of finite type over $\bar{X}$. Let $\pi_{1}$ be the projection from $\bar{X}$ to $X$, and let $\pi_{2}$ be the projection from $\bar{X}$ to $\bar{k}$. If $(W, f: W \rightarrow \bar{X})$ and $(Z, g: Z \rightarrow \bar{X})$ are objects in Cat $(\mathcal{W})$ with $W$ connected, a morphism $\phi$ from $(W, f)$ to $(Z, g)$ is a morphism $\phi$ from $W$ to $Z$ such that

(a) $\pi_{1} g \phi=\pi_{1} f$ and

(b) there exists an integer $n$ such that $\pi_{2} g \phi=\pi_{2} f(\text { Frob })^{n}$, where (Frob) is the Frobenius morphism from $\operatorname{Spec} \bar{k}$ to itself.

(Note that for once we do not have to worry about whether we are using the arithmetic or geometric Frobenius.) A morphism from an arbitrary $W$ is a collection of morphisms on the connected components of $W$. The coverings are the surjective families $\left\{W_{i} \rightarrow Z\right\}$ in $\operatorname{Cat}(\mathcal{W})$, i.e. obtained by restricting the étale topology from the small étale site of $\bar{X}$ to the subcategory $\operatorname{Cat}(\mathcal{W})$.

We recall that the Galois group of $\bar{k}$ over $k$ is naturally isomorphic to $\Gamma$, and we let $\Gamma_{0}$ be the subgroup of $\Gamma$ consisting of the integral powers of Frobenius. If $G$ is a Weil-étale sheaf on $X$ then $G(\bar{X})$ is in a natural way a $\Gamma_{0}$-module. We define $H_{\mathcal{W}}^{0}(X, G)$ to be $G(\bar{X})^{\Gamma_{0}}$, and $H_{\mathcal{W}}^{i}(X, G)$ to be the derived functors of $H_{\mathcal{W}}^{0}$.

Definition 2.1. Let $H$ be a group of automorphisms of a scheme $X$. We say that $H$ acts on a sheaf $F$ on $X$ if we have a compatible system of maps $\psi_{\sigma}: F \rightarrow \sigma_{*} F$ for all $\sigma$ in $H$.

Proposition 2.2. The category of Weil-étale sheaves on $X$ is equivalent to the category of étale sheaves on $\bar{X}$ equipped with a $\Gamma_{0}$-action.

Proof. A Weil-étale sheaf $G$ on $X$ restricts to an étale sheaf $\rho(G)$ on $\bar{X}$. If $U$ is an étale scheme over $\bar{X}$, and $\sigma$ is in $\Gamma_{0}$, let $U_{\sigma}=U \times_{\bar{X}} \bar{X}$, where the map from $\bar{X}$ to $\bar{X}$ is given by $\sigma$. Then the projection map from $U_{\sigma}$ to $U$ is a map in our category, and so determines a functorial map $G(U)$ to $G\left(U_{\sigma}\right)$, which is exactly a map from $\rho(G)$ to $\sigma_{*} \rho(G)$. 


\section{S. LiCHTENBAUM}

To go in the other direction, a map in our category from $V$ to $W$ gives rise to a commutative diagram

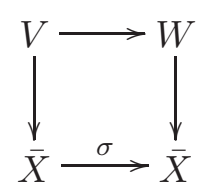

where $\sigma$ is in $\Gamma_{0}$. This clearly determines an $\bar{X}$-map from $V$ to $W_{\sigma}$, and thus a map $G(W) \rightarrow$ $G\left(W_{\sigma}\right) \rightarrow G(V)$, where the map from $G(W)$ to $G\left(W_{\sigma}\right)$ is given by $\left(\psi_{\sigma}\right)^{*}$.

The subcategory of lisse sheaves on $\bar{X}$ with a $\Gamma_{0}$-action is equivalent to the category of 'Weil sheaves' introduced by Deligne in [Del80], but Deligne did not actually define a topology, nor consider sheaves of the type we work with here.

Proposition 2.3. Let $G$ be a Weil-étale sheaf on $X$. There is a spectral sequence whose $E_{2}^{p, q}$-term is $H^{p}\left(\Gamma_{0}, H_{\text {ét }}^{q}(\bar{X}, \rho(G))\right)$ and which converges to $H_{\mathcal{W}}^{p+q}(X, G)$.

Proof. The category of sheaves for the Weil-étale site on $k$ is equivalent to the category of $\Gamma_{0}$-modules and the cohomology groups $H_{W}^{i}(k, G)$ are canonically isomorphic to the cohomology groups $H^{i}\left(\Gamma_{0}, G(\bar{k})\right)$. The functor $\left(\pi_{2}\right)_{*}$ has the exact left adjoint $\pi_{2}^{*}$, and so takes injectives to injectives. Our spectral sequence now just becomes a usual composite functor spectral sequence.

We define a pair of functors: $\psi$, which maps Weil-étale sheaves on $X$ to étale sheaves on $X$, and $\phi$, which maps étale sheaves on $X$ to Weil-étale sheaves on $X$. We define $\psi$ as follows: if $G$ is a Weil-étale sheaf, define $\psi(G)(U)$ to be $\left(G\left(U \times_{X} \bar{X}\right)\right)^{\Gamma_{0}}$. If $F$ is an étale sheaf on $X$, define $\phi(F)$ to be $\pi_{1}^{*}(F)$ (which is endowed with a natural $\Gamma_{0}$-action).

It is readily verified that these functors have the properties listed in the following proposition.

Proposition 2.4.

(a) $\phi$ is left adjoint to $\psi$.

(b) $\psi \phi=1$.

(c) $\psi$ is left exact and $\phi$ is exact, so $\psi$ takes injectives to injectives.

(d) $\psi(\mathbf{Z})=\mathbf{Z}$, and $\psi(\mathbf{Z} / n \mathbf{Z})=\mathbf{Z} / n \mathbf{Z}$.

(e) There is a functorial map $c_{i}: H_{\text {ét }}^{i}(X, \psi(G)) \rightarrow H_{\mathcal{W}}^{i}(X, G)$ which is an isomorphism when $i=0$, and, by (b), a functorial map $d_{i}: H_{\text {ét }}^{i}(X, F) \rightarrow H_{\mathcal{W}}^{i}(X, \phi(F))$.

(f) Let $F$ be an étale sheaf on $X$. There is a functorial map of spectral sequences from

$$
H^{p}\left(\Gamma, H_{\text {ét }}^{q}\left(\bar{X}, \pi_{1}^{*} F\right)\right) \Rightarrow H_{\text {ét }}^{p+q}(X, F)
$$

to

$$
H^{p}\left(\Gamma_{0}, H_{\text {ét }}^{q}(\bar{X}, \rho \phi(F))\right) \Rightarrow H_{\mathcal{W}}^{p+q}(X, \phi(F)) .
$$

Of course, this last spectral sequence breaks up into short exact sequences:

$$
0 \rightarrow H^{1}\left(\Gamma_{0}, H_{\text {ét }}^{q}(\bar{X}, \rho \phi(F))\right) \rightarrow H_{\mathcal{W}}^{q+1}(X, \phi(F)) \rightarrow H^{0}\left(\Gamma_{0}, H_{\text {ét }}^{q+1}(\bar{X}, \rho \phi(F))\right) \rightarrow 0 .
$$

(g) $c_{i}$ and $d_{i}$ are isomorphisms if $G$ is torsion.

Note that in Proposition 2.4 part (f), the first spectral sequence is the standard Hochschild-Serre spectral sequence and the second is the one from Proposition 2.3, and the fact that $\rho \phi(F)=\pi_{1}^{*}(F)$. Proposition 2.4 part (g) then follows from a comparison of these spectral sequences, using Lemma 1.1. 


\section{The WeIL-ÉtAle TOPOLOGY ON SCHEMES OVER FINITE FIELDS}

We remark that, since we are in the 'small-site' Weil-étale topology, the apparatus of the mapping cylinder category (see [Mil80, pp. 73-77]) goes through without change. In particular, if $Z$ is a closed subscheme of $X$ with open complement $U$, and $i$ and $j$ are the immersions from $Z$ and $U$ into $X$, we have the six standard functors $i^{*}, i_{*}, i^{!}, j_{!}, j^{*}$, and $j_{*}$, with the standard adjointness relations ( $i_{*}$ is right adjoint to $i^{*}$ and left adjoint to $i^{!}, j^{*}$ is right adjoint to $j_{\text {! }}$ and left adjoint to $j_{*}$ ), and satisfying $i^{*} i_{*}=\mathrm{id}$, and $i^{*} j_{!}=0$. If $X$ is a smooth curve over a finite field $k$ with field of fractions $F$, and $\phi:$ Spec $F \rightarrow X$ is the induced map, we have the exact sequence

$$
0 \rightarrow \mathbb{G}_{m, X} \rightarrow \phi_{*} \mathbb{G}_{m, F} \rightarrow \coprod\left(i_{x}\right)_{*} \mathbf{Z} \rightarrow 0,
$$

from which it follows that $R^{q} i^{!} \mathbb{G}_{m, X}=0$ for $q \neq 1$ and $R^{1} i^{!} \mathbb{G}_{m, X}=\mathbf{Z}$.

\section{The finite generation of cohomology groups}

Throughout this section let $k$ be a fixed finite field.

Theorem 3.1. Let $X$ be a projective smooth variety over $k$. The Weil-étale cohomology groups $H_{\mathcal{W}}^{q}(X, \mathbf{Z})$ are finitely generated for all $q$, finite for $q \geqslant 2$, and zero for $q$ large.

Proof. We may assume $X$ connected. It follows from [Mil86] that $H_{\text {ét }}^{q}(X, \mathbf{Z})$ is finite for $q \geqslant 3$ and zero for $q$ large and for $q=1$, that $H_{\text {ét }}^{2}(X, \mathbf{Z})$ is the $\mathbf{Q} / \mathbf{Z}$-dual of a finitely generated group of rank 1 (so isomorphic to the direct sum of $\mathbf{Q} / \mathbf{Z}$ with a finite abelian group $A$ ), and of course $H_{\text {ét }}^{0}(X, \mathbf{Z})=\mathbf{Z}$. We observe that, if we let $\bar{K}$ be the fraction field of $\bar{X}$ and $\bar{j}:$ Spec $\bar{K} \rightarrow \bar{X}$, then the constant sheaf $\mathbf{Z}$ on $\bar{X}$ is the direct image of the constant sheaf $\mathbf{Z}$ on Spec $\bar{K}$. The Leray spectral sequence for $\bar{j}$ shows then that $H^{q}(\bar{X}, \mathbf{Z})$ is torsion for $q>0$, since Galois cohomology is always torsion.

A comparison of the spectral sequences given at the end of the preceding section then shows that $H_{\mathcal{W}}^{q}(X, \mathbf{Z})$ is canonically isomorphic to $H_{\text {ét }}^{q}(X, \mathbf{Z})$ (and hence finite and zero for $q$ large) for $q \geqslant 3$, and that $H_{\mathcal{W}}^{2}(X, \mathbf{Z})$ is isomorphic to $A=H_{\text {ét }}^{2}(X, \mathbf{Z}) /(\mathbf{Q} / \mathbf{Z})$ and hence finite. It also follows immediately that $H_{\mathcal{W}}^{0}(X, \mathbf{Z})$ and $H_{\mathcal{W}}^{1}(X, \mathbf{Z})$ are both isomorphic to $\mathbf{Z}$.

Lemma 3.2. Let $U$ be a curve over the finite field $k$. Let $F$ be any Weil-étale sheaf on $U$. Let $j$ : $U \rightarrow X$ embed $U$ as a dense open subset of a projective curve $X$. Then

(a) the groups $H_{\mathcal{W}}^{q}(X, j ! F)$ are independent of $j$ and

(b) if $F=\mathbf{Z}$, then they are finitely generated.

Proof. (a) Let $j: U \rightarrow X$ and $j^{\prime}: U \rightarrow X^{\prime}$ be two completions of $U$. Replacing $X^{\prime}$ by the closure of the image of $U$ in $X \times X^{\prime}$ we may assume that there is a map $\pi: X^{\prime} \rightarrow X$ such that $\pi \circ j^{\prime}=j$. Since $\pi$ is finite, so is $\bar{\pi}$, and $\bar{\pi}_{*}$ is exact in the étale topology. It follows that the natural map from $H_{\text {ét }}^{q}\left(\bar{X}, \bar{\pi}_{*} F\right)$ to $H_{\text {ét }}^{q}\left(\bar{X}^{\prime}, \bar{F}\right)$ is an isomorphism, and hence, by the basic spectral sequence of Proposition 2.3, the natural map from $H_{\mathcal{W}}^{q}\left(X, \pi_{*} F\right)$ to $H_{\mathcal{W}}^{q}\left(X^{\prime}, F\right)$ is an isomorphism. But $\pi_{*} j_{!} F$ is naturally isomorphic to $j_{!}^{\prime} F$ (construct a map, and then verify that it is an isomorphism on stalks), and so the cohomology groups $H_{\mathcal{W}}^{q}\left(X^{\prime}, j_{!}^{\prime} F\right)$ are isomorphic to the groups $H_{\mathcal{W}}^{q}(X, j ! F)$.

(b) Let $U$ be an open dense subscheme of $V$ which in turn is an open dense subscheme of the projective curve $X$. Let $\phi: U \rightarrow X$ and $j: V \rightarrow X$ be the given open immersions, and $i$ the closed immersion of $V-U$ in $X$. We have the exact sequence

$$
0 \rightarrow \phi_{!} \mathbf{Z} \rightarrow j ! \mathbf{Z} \rightarrow i_{*} \mathbf{Z} \rightarrow 0
$$

Then part (b) follows from the consideration of the related long exact cohomology sequence, showing that first the cohomology groups of smooth projective curves, then smooth curves, and then arbitrary curves, are finitely generated. 


\section{S. LichtenBAUM}

Theorem 3.3. Let $d \leqslant 2$ and let $U$ be a smooth $d$-dimensional quasi-projective variety over $k$. $B y$ resolution of singularities we can find a smooth projective variety $X$ containing $U$ as an open dense subvariety. Let $j: U \rightarrow X$ be the corresponding open immersion. Then the cohomology groups $H_{\mathcal{W}}^{q}(X, j ! \mathbf{Z})$ are finitely generated, zero for $q$ large, and independent of the choices of $X$ and $j$.

Proof. We write $H^{q}$ for $H_{\mathcal{W}}^{q}$. We first show finite generation. Let $j: U \rightarrow X$ as above, and let $Z=X-U$. Let $i: Z \rightarrow X$ be the corresponding closed immersion. We have the exact sequence of sheaves on $X: 0 \rightarrow j ! \mathbf{Z} \rightarrow \mathbf{Z} \rightarrow i_{*} \mathbf{Z} \rightarrow 0$. Since $i_{*}$ is exact, Theorem 3.1 implies that the cohomology groups $H^{q}\left(X, i_{*} \mathbf{Z}\right) \stackrel{\sim}{\rightarrow} H^{q}(Z, \mathbf{Z})$ are finitely generated and zero for $q$ large, and Lemma 3.2 implies that the cohomology groups $H^{q}(X, \mathbf{Z})$ are finitely generated and zero for $q$ large. Hence the long exact cohomology sequence implies that the groups $H^{q}\left(X, j_{!} \mathbf{Z}\right)$ are also finitely generated and zero for $q$ large.

Now we show independence. Suppose we have $j: U \rightarrow X$ and $j^{\prime}: U \rightarrow W$ with $X$ and $W$ being smooth projective. Let $\rho: U \rightarrow V=X \times W$ be the map induced by $j$ and $j^{\prime}$, and let $Z$ be the closure of $U$ in $V$. By resolution we can find a $\pi: Z^{\prime} \rightarrow Z$ such that $Z^{\prime}$ is projective and smooth. Replacing $W$ by $Z^{\prime}$, we may assume that there is a map $\pi: W \rightarrow X$ such that $\pi \circ j^{\prime}=j$. We next observe that $\pi_{*} j_{!}^{\prime} \mathbf{Z}=j_{!} \mathbf{Z}$, and then the functorial map from $H^{q}\left(X, \pi_{*} F\right)$ to $H^{q}(W, F)$ gives us the following commutative diagram:

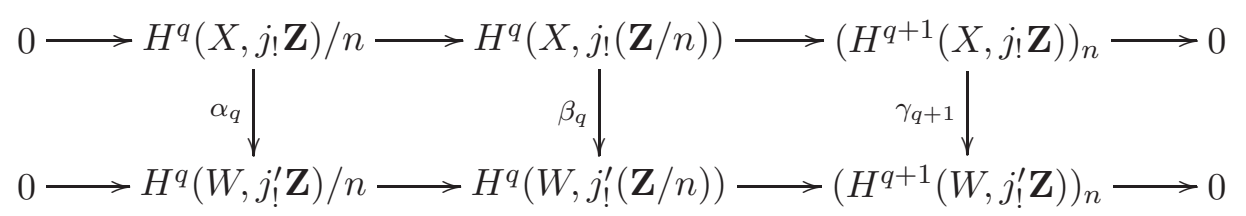

We know that $\beta$ is an isomorphism because the standard étale cohomology groups with compact support of constructible sheaves are well defined. We proceed by descending induction. Let $\delta_{q}$ : $H^{q}\left(X, j_{!} \mathbf{Z}\right) \rightarrow H^{q}\left(W, j_{!}^{\prime} \mathbf{Z}\right)$. If $\delta_{q+1}$ is an isomorphism then $\gamma_{q+1}$ is an isomorphism, hence $\alpha_{q}$ is an isomorphism. Now if we have a map $f$ from one finitely generated group to another such that $f$ becomes an isomorphism after tensoring with $\mathbf{Z} / n$ for every $n$, then it is easy to see that $f$ must be an isomorphism. So $\delta_{q}$ is an isomorphism. We can start the induction because both groups $H^{q}$ are zero for large $q$.

In a slight abuse of notation, let $\mathbb{G}_{m}$ be the Weil-étale sheaf obtained by restricting the étale sheaf $\mathbb{G}_{m}$ to the Weil-étale site.

Proposition 3.4. Let $X$ be a geometrically connected smooth curve over a finite field $k$. Then the cohomology groups $H_{\mathcal{W}}^{q}\left(X, \mathbb{G}_{m}\right)$ are finitely generated for all $q$ and zero if $q \geqslant 3$. If $X$ is projective, $H_{\mathcal{W}}^{0}\left(X, \mathbb{G}_{m}\right)=k^{*}$ and $H_{\mathcal{W}}^{1}\left(X, \mathbb{G}_{m}\right)=\operatorname{Pic}(X)$ just as in the étale case, and $H_{\mathcal{W}}^{2}\left(X, \mathbb{G}_{m}\right)=\mathbf{Z}$.

Proof. We begin with the spectral sequence of Proposition 2.3:

$$
H^{p}\left(\Gamma_{0}, H_{\text {ét }}^{q}\left(\bar{X}, \mathbb{G}_{m}\right)\right) \Rightarrow H_{\mathcal{W}}^{p+q}\left(X, \mathbb{G}_{m}\right)
$$

This spectral sequence degenerates to give the series of short exact sequences

$$
0 \rightarrow H^{1}\left(\Gamma_{0}, H_{\text {ét }}^{q-1}\left(\bar{X}, \mathbb{G}_{m}\right)\right) \rightarrow H_{\mathcal{W}}^{q}\left(X, \mathbb{G}_{m}\right) \rightarrow H^{0}\left(\Gamma_{0}, H_{\text {ét }}^{q}\left(\bar{X}, \mathbb{G}_{m}\right)\right) \rightarrow 0 .
$$

We now plug in the fact [Mil80, Example 2.22d] that $H_{\text {ét }}^{q}\left(\bar{X}, \mathbb{G}_{m}\right)=0$ for $q \geqslant 2$. We immediately obtain $H_{\mathcal{W}}^{q}\left(X, \mathbb{G}_{m}\right)=0$ for $q \geqslant 3$.

We also see that $H_{\mathcal{W}}^{2}\left(X, \mathbb{G}_{m}\right)$ is isomorphic to $H^{1}\left(\Gamma_{0}, \operatorname{Pic}(\bar{X})\right)$. Let $U(\bar{X})=H^{0}\left(\bar{X}, \mathbb{G}_{m}\right)$. We have an exact sequence

$$
0 \rightarrow H^{1}\left(\Gamma_{0}, U(\bar{X})\right) \rightarrow H_{\mathcal{W}}^{1}\left(X, \mathbb{G}_{m}\right) \rightarrow(\operatorname{Pic}(\bar{X}))^{\Gamma_{0}} \rightarrow 0
$$




\section{The WeIL-ÉtAle TOPOLOGY ON SCHEMES OVER FINITE FIELDS}

Now write $X$ as $Y-S$ with $Y$ smooth and projective and $S$ finite. Let $T$ be the finite set of points of $\bar{Y}$ lying over $S$. First assume that $S$ is empty. Since $(\operatorname{Div}(\bar{Y}))^{\Gamma_{0}}=\operatorname{Div}(Y)$, it follows that the cokernel of the map from $\operatorname{Pic}(Y)$ to $(\operatorname{Pic}(\bar{Y}))^{\Gamma_{0}}$ is contained in $H^{1}\left(\Gamma_{0}, k(\bar{Y})^{*} /(\bar{k})^{*}\right)$, which is contained in $H^{2}\left(\Gamma_{0},(\bar{k})^{*}\right)$ by Hilbert's Theorem 90 . Then $(\operatorname{Pic}(\bar{Y}))^{\Gamma_{0}} \stackrel{\sim}{\rightarrow} \operatorname{Pic}(Y)$ since $H^{2}\left(\Gamma_{0}, \bar{k}^{*}\right)=0$. Since $H^{1}\left(\Gamma_{0}, \operatorname{Pic}_{0}(\bar{Y})\right)=0$ by Lang's theorem (any principal homogeneous space of a connected algebraic group over a finite field is trivial; see [Ser59, pp. 112 and 119$])$, then $H^{1}\left(\Gamma_{0}, \operatorname{Pic}(\bar{Y})\right) \stackrel{\sim}{\rightarrow}$ $H^{1}\left(\Gamma_{0}, \mathbf{Z}\right) \stackrel{\sim}{\longrightarrow}$. The statement about $H^{0}$ is clear.

Now let $X$ be arbitrary. We have $U(\bar{Y})=\bar{k}^{*}$ and the exact sequences:

$$
\begin{gathered}
0 \rightarrow U(\bar{Y}) \rightarrow U(\bar{Y}-T) \rightarrow M \rightarrow 0, \\
0 \rightarrow N \rightarrow \operatorname{Pic}(\bar{Y}) \rightarrow \operatorname{Pic}(\bar{Y}-T) \rightarrow 0,
\end{gathered}
$$

where $M$ and $N$ are $\Gamma_{0}$-modules which are finitely generated as abelian groups and so whose $\Gamma_{0}$-cohomology is also finitely generated. Since $H^{q}\left(\Gamma_{0}, \bar{k}^{*}\right)$ and $H^{q}\left(\Gamma_{0}, \operatorname{Pic}(\bar{Y})\right)$ are both finitely generated for all $q$, the result follows. (Since $\Gamma_{0}$ has cohomological dimension 1, we need only look at $q=0$ and $q=1$. The fact that $H^{1}\left(\Gamma_{0}, \bar{k}^{*}\right)=0$ follows from the equality of $\Gamma_{0}$-cohomology with $\Gamma$-cohomology for torsion coefficients and Hilbert's Theorem 90. The finite generation of $H^{1}\left(\Gamma_{0}, \operatorname{Pic}(\bar{Y})\right)$ again follows from Lang's theorem. When $q=0$, the result is evident.)

\section{Verdier duality for abelian groups}

In this section we state the surely well-known derived category version of duality in the category of abelian groups. Let $\mathcal{D}$ be the full subcategory of the bounded derived category $D^{b}(\mathbf{Z})$ of abelian groups consisting of those complexes with finitely generated homology groups. If $A$ is in $\mathcal{D}$, let $A^{*}$ be $R \operatorname{Hom}(A, \mathbf{Z})$. Since $\mathbf{Z}$ has finite injective dimension, $A^{*}$ is again in $\mathcal{D}$.

We recall Theorem 10.8.7 of [Wei94]: If $R$ is a commutative ring and $B$ is a bounded above complex of $R$-modules, then $\otimes_{R}^{L} B: D^{-}(R) \rightarrow D^{-}(R)$ is left adjoint to the functor $R \operatorname{Hom}_{R}(B,-)$ : $D^{+}(R) \rightarrow D^{+}(R)$. That is, for $A$ in $D^{-}(R)$ and $C$ in $D^{+}(R)$ there is a natural isomorphism:

$$
\operatorname{Hom}_{D(R)}\left(A, R \operatorname{Hom}_{R}(B, C)\right) \stackrel{\sim}{\rightarrow} \operatorname{Hom}_{D(R)}\left(A \otimes_{R}^{L} B, C\right) .
$$

Now let $R=\mathbf{Z}$, let $A$ be in $\mathcal{D}, B=A^{*}$, and $C=\mathbf{Z}$. By applying Weibel's Theorem 10.8.7 twice, we obtain

$$
\operatorname{Hom}_{\mathcal{D}}\left(A, R \operatorname{Hom}\left(A^{*}, \mathbf{Z}\right)\right) \stackrel{\sim}{\rightarrow} \operatorname{Hom}_{\mathcal{D}}\left(A \otimes A^{*}, \mathbf{Z}\right) \stackrel{\sim}{\rightarrow} \operatorname{Hom}_{\mathcal{D}}\left(A^{*} \otimes A, \mathbf{Z}\right) \stackrel{\sim}{\rightarrow} \operatorname{Hom}_{\mathcal{D}}\left(A^{*}, A^{*}\right),
$$

so there is a canonical map $\alpha$ in the derived category from $A$ to $A^{* *}$, corresponding to the identity in $\operatorname{Hom}_{\mathcal{D}}\left(A^{*}, A^{*}\right)$.

Proposition 4.1. The map $\alpha$ is an isomorphism in $\mathcal{D}$; hence the functor $R \operatorname{Hom}(-, \mathbf{Z})$ is a contravariant equivalence of categories which is inverse to itself.

Proof. The proof is by an easy induction on the length $n$ of the complex $A$. If $n=1$ it follows immediately, because if $F$ is free and finitely generated, then $\operatorname{Hom}(F, \mathbf{Z})$ is dualizing, and if $M$ is finite, then $\operatorname{Ext}^{1}(M, \mathbf{Z}) \stackrel{\sim}{\rightarrow} \operatorname{Hom}(M, \mathbf{Q} / \mathbf{Z})$ is dualizing.

\section{The duality theorem for non-singular curves}

Let $U$ be a smooth geometrically connected curve over a finite field $k$, let $\mathcal{S} h_{U}$ be the abelian category of Weil-étale sheaves on $U$, and let $\Gamma_{U}(F)=H_{\mathcal{W}}^{0}(U, F)$. Let $j: U \rightarrow X$ be an open dense embedding of $U$ in a smooth projective curve $X$ over $k$, and let $F$ be an object of $\mathcal{S} h_{X}$. Since $X$ has finite cohomological dimension, we may define $R \Gamma_{X}(F)$ as an element of the bounded derived category 


\section{S. Lichtenbaum}

by taking an injective resolution $I^{\bullet}$ of $F$, taking a sufficiently far-out truncation $\tau I^{\bullet}$, and applying the functor $\Gamma_{X}$. It is immediate that this defines a unique element in the bounded derived category of abelian groups. Let $J^{\bullet}$ be an injective resolution of $\mathbb{G}_{m}$. Then applying the functor $\Gamma_{X}$ induces a natural map in $D(\mathbf{Z})$ from $R \operatorname{Hom}_{X}\left(F, \mathbb{G}_{m}\right)=\operatorname{Hom}_{X}\left(\tau I^{\bullet}, J^{\bullet}\right)$ to $R \operatorname{Hom}_{D(\mathbf{Z})}\left(R \Gamma_{X}(F), R \Gamma_{X}\left(\mathbb{G}_{m}\right)\right)$.

Recall from Proposition 3.4 that $H_{\mathcal{W}}^{2}\left(X, \mathbb{G}_{m}\right)=\mathbf{Z}$ and $H_{\mathcal{W}}^{q}\left(X, \mathbb{G}_{m}\right)=0$ for $q \geqslant 3$, which gives us a natural map in $D(\mathbf{Z})$ from $R \Gamma_{X}\left(\mathbb{G}_{m}\right)$ to $\mathbf{Z}[-2]$. Now compose with the above map to get a map $\kappa_{F}$ from $R \operatorname{Hom}_{X}\left(F, \mathbb{G}_{m}\right)$ to $R \operatorname{Hom}_{D(\mathbf{Z})}\left(R \Gamma_{X}(F), \mathbf{Z}[-2]\right)$.

Theorem 5.1. Let $F$ be either $j ! \mathbf{Z}$ (in which case we denote $\kappa_{F}$ by $\kappa$ ) or $j ! \mathbf{Z} / n \mathbf{Z}$ (in which case we denote $\kappa_{F}$ by $\kappa_{n}$ ). Then $\kappa_{F}$ is an isomorphism.

Proof. We begin with the case $F=j ! \mathbf{Z} / n \mathbf{Z}$. Let $J_{n}$ denote $j ! \mathbf{Z} / n \mathbf{Z}$ (as both étale and Weil-étale sheaf). We have the commutative diagram in the derived category of abelian groups:

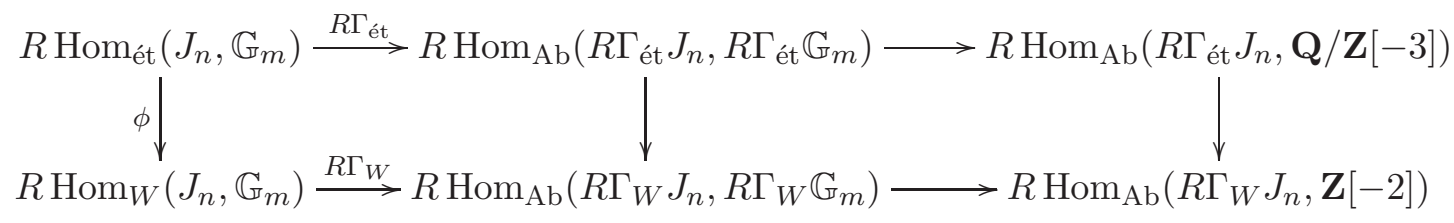

The composite of the two top horizontal arrows is the duality isomorphism interpreting class field theory for function fields in terms of étale cohomology, which was proved by Deninger in [Den84]. (If $F$ is a constructible torsion sheaf on $X$, then we have a map induced by $R \Gamma$ from $C_{1}=$ $R \operatorname{Hom}_{\text {ét }}\left(F, \mathbb{G}_{m}\right)$ to $R \operatorname{Hom}\left(R \Gamma F, R \Gamma \mathbb{G}_{m}\right)$ to $R \operatorname{Hom}(R \Gamma F, \mathbf{Q} / \mathbf{Z}[-3])=C_{2}$, since $H^{i}\left(X, \mathbb{G}_{m}\right)=0$ for $i>3$, and $H^{3}\left(X, \mathbb{G}_{m}\right)=\mathbf{Q} / \mathbf{Z}[-3]$. The homology of $C_{1}$ is $\operatorname{Ext}_{X}^{i}\left(F, \mathbb{G}_{m}\right)$, and the homology of $C_{2}$ is $\left(H^{3-i}(X, F)\right)^{*}$, where $*$ denotes Pontryagin dual. The induced maps on homology come from the Yoneda pairing. Deninger shows [Den84, Theorem 1.7, p. 94] that these maps are isomorphisms, and hence it follows that the map from $C_{1}$ to $C_{2}$ is a quasi-isomorphism, so an isomorphism in the derived category.) What we want to show is that the composite of the two bottom horizontal arrows is an isomorphism, so it suffices to show that the first and last vertical arrows are isomorphisms.

The last vertical arrow is an isomorphism because $\phi$ induces an isomorphism between $R \Gamma_{\text {ét }} J_{n}$ and $R \Gamma_{W} J_{n}$, and $R \operatorname{Hom}_{\mathrm{Ab}}\left(R \Gamma_{\text {ét }} J_{n}, \mathbf{Q}\right)=0\left(\operatorname{Ext}^{i}(A, \mathbf{Q})=0\right.$ if $A$ is killed by $\left.n\right)$.

We now wish to show that the first vertical arrow is an isomorphism. Let $Z$ be the closed complement of $U$, with the induced reduced structure, and let $i: Z \rightarrow X$ be the natural inclusion. Let $F$ be a sheaf on $X$. In both the étale and Weil-étale topologies, $\underline{\operatorname{Hom}}\left(i_{*} \mathbf{Z}, F\right)$ is naturally isomorphic to $i^{!} F$, and hence $\underline{\operatorname{Ext}}^{q}\left(i_{*} \mathbf{Z}, F\right)$ is naturally isomorphic to $R^{q} i ! F$. In particular, if $F=\mathbb{G}_{m}$ we have that $\underline{\operatorname{Ext}}^{q}\left(i_{*} \mathbf{Z}, \mathbb{G}_{m}\right)$ is naturally isomorphic to $R^{q} i \mathbb{G}_{m}$, which is zero if $q \neq 1$ and $\mathbf{Z}$ if $q=1$ (see the remarks at the end of $\S 2$ ). Hence $\phi$ takes the étale version of $\operatorname{Ext}^{q}\left(i_{*} \mathbf{Z}, \mathbb{G}_{m}\right)$ to the Weil-étale version of the same.

Since $\underline{\operatorname{Ext}}^{q}\left(\mathbf{Z}, \mathbb{G}_{m}\right)$ equals $\mathbb{G}_{m}$ if $q=0$ and 0 if $q \neq 0, \phi$ also preserves it, and the exact sequence

$$
0 \rightarrow j ! \mathbf{Z} \rightarrow \mathbf{Z} \rightarrow i_{*} \mathbf{Z} \rightarrow 0
$$

shows that $\phi$ preserves $\underline{\operatorname{Ext}}^{q}\left(j ! \mathbf{Z}, \mathbb{G}_{m}\right)$ as well. It then immediately follows that $\phi$ also preserves $\underline{\operatorname{Ext}}^{q}\left(J_{n}, \mathbb{G}_{m}\right)$.

Now the local to global spectral sequence, and the fact that $J_{n}$ is killed by $n$, shows that $\phi$ also preserves $\operatorname{Ext}^{q}\left(J_{n}, \mathbb{G}_{m}\right)$, and hence $R \operatorname{Hom}\left(J_{n}, \mathbb{G}_{m}\right)$, which is what was wanted.

So we have shown that $\kappa_{n}$ is an isomorphism, and we want to complete the proof of Theorem 5.1 by showing that $\kappa$ is an isomorphism.

By a straightforward descending induction, we obtain the following lemma. 


\section{The WeIL-ÉtAle TOPOLOGY ON SCHEMES OVER FINITE FIELDS}

Lemma 5.2. Let $M^{\bullet}$ and $N^{\bullet}$ be complexes of abelian groups and $g$ a map from $M^{\bullet}$ to $N^{\bullet}$. Assume further that there are complexes $M_{n}^{\cdot}$ and $N_{n}^{*}$ for every positive integer $n$ such that the following hold.

(1) There is a map $a_{n}$ from $M^{\bullet}$ to $M_{n}^{\cdot}$ such that there exists a triangle in the derived category of abelian groups $M^{\bullet} \stackrel{n}{\rightarrow} M^{\bullet} \stackrel{a_{n}}{\rightarrow} M_{n}^{\bullet} \rightarrow M^{\bullet}[1]$.

(2) There is a map $b_{n}$ from $N^{\bullet}$ to $N_{n}^{\bullet}$ such that there exists a triangle in the derived category of abelian groups $N^{\bullet} \stackrel{n}{\rightarrow} N^{\bullet} \stackrel{b_{n}}{\rightarrow} N_{n}^{\bullet} \rightarrow N^{\bullet}[1]$.

(3) There is a quasi-isomorphism $g_{n}$ from $M_{n}^{\bullet}$ to $N_{n}^{\cdot}$ such that the triple $g, g, g_{n}$ induces a map of the aforementioned triangles.

(4) The homology groups $h^{i}\left(M^{\bullet}\right)$ and $h^{i}\left(N^{\bullet}\right)$ are finitely generated for all $i$ and zero for $i$ large.

Then $g$ is a quasi-isomorphism.

Lemma 5.3. Let $G$ be a Weil-étale sheaf on $U$. There is a canonical isomorphism in $D(\mathbf{Z})$ between $R \operatorname{Hom}_{X}\left(j ! G, \mathbb{G}_{m, X}\right)$ and $R \operatorname{Hom}_{U}\left(G, \mathbb{G}_{m, U}\right)$.

Proof. Because $j^{*}$ has the exact left adjoint $j !, j^{*}$ takes injectives to injectives. Since $j^{*}$ is exact, it carries a resolution of $\mathbb{G}_{m, X}$ to a resolution of $j^{*} \mathbb{G}_{m, X}=\mathbb{G}_{m, U}$. The isomorphism then follows immediately from the adjointness of $j^{*}$ and $j$ !

To complete the proof of Theorem 5.1, we need only point out that the cohomology groups in question are finitely generated and zero for large $i$ by Proposition 3.4 and Lemma 5.3, and then apply Lemma 5.2.

Theorem 5.4. (a) $R \Gamma_{U}\left(\mathbb{G}_{m}\right)$ is naturally isomorphic to $R \operatorname{Hom}_{D(\mathbf{Z})}\left(R \Gamma_{X}(j ! \mathbf{Z}), \mathbf{Z}[-2]\right)$.

(b) $R \Gamma_{X}(j ! \mathbf{Z})$ is naturally isomorphic to $R \operatorname{Hom}_{D(\mathbf{Z})}\left(R \Gamma_{U}\left(\mathbb{G}_{m}\right), \mathbf{Z}[-2]\right)$.

Proof. Since $R \Gamma_{U}(F)$ is the same as $R \operatorname{Hom}_{U}(\mathbf{Z}, F)$, part (a) follows immediately from Theorem 5.1 and Lemma 5.3. Since the functor $R \operatorname{Hom}(\cdot, \mathbf{Z}[-2])$ is its own inverse, part (b) follows from part (a).

What all this means in terms of cohomology groups is spelled out in the next section.

\section{The computation of some cohomology groups}

In this section we will compute the Weil-étale cohomology of $\mathbb{G}_{m}$ and $j ! \mathbf{Z}$ on a smooth curve $V$.

TheOREM 6.1. Let $X$ be a projective smooth geometrically connected curve over the finite field $k$. Then we have the following:

(a) $H_{\mathcal{W}}^{0}\left(X, \mathbb{G}_{m}\right)=k^{*}, H_{\mathcal{W}}^{1}\left(X, \mathbb{G}_{m}\right)=\operatorname{Pic}(X), H_{\mathcal{W}}^{2}\left(X, \mathbb{G}_{m}\right)=\mathbf{Z}$, and $H_{\mathcal{W}}^{i}\left(X, \mathbb{G}_{m}\right)=0$ for $i \geqslant 3$.

(b) $H_{\mathcal{W}}^{0}(X, \mathbf{Z})=\mathbf{Z}, H_{\mathcal{W}}^{1}(X, \mathbf{Z})=\operatorname{Hom}(\operatorname{Pic}(X), \mathbf{Z})=\mathbf{Z}, H_{\mathcal{W}}^{2}(X, \mathbf{Z})=\operatorname{Ext}(\operatorname{Pic}(X), \mathbf{Z})$, which is the Pontryagin dual of the finite group $\operatorname{Pic}_{0}(X), H_{\mathcal{W}}^{3}(X, \mathbf{Z})=\operatorname{Ext}\left(k^{*}, \mathbf{Z}\right)$, which is the Pontryagin dual of the finite group $k^{*}$, and $H_{\mathcal{W}}^{i}(X, \mathbf{Z})=0$ for $i \geqslant 4$.

Let $V=X-S$ be a quasi-projective smooth curve over the finite field $k$, with $X$ projective, smooth and geometrically connected, and $S$ finite and non-empty. Let $j: V \rightarrow X$ be the natural open immersion and $i: S \rightarrow X$ be the natural closed immersion. Then we have the following:

(c) $H_{\mathcal{W}}^{0}\left(V, \mathbb{G}_{m}\right)=U(V)=$ the units of $V$. We have the exact sequence

$$
0 \rightarrow \operatorname{Pic}(V) \rightarrow H_{\mathcal{W}}^{1}\left(V, \mathbb{G}_{m}\right) \rightarrow \operatorname{Hom}\left(\coprod_{S} \mathbf{Z} / \mathbf{Z}, \mathbf{Z}\right) \rightarrow 0
$$

and $H_{\mathcal{W}}^{i}\left(V, \mathbb{G}_{m}\right)=0$ for $i \geqslant 2$. 


\section{S. LichtenBAum}

(d) $H_{\mathcal{W}}^{0}(X, j ! \mathbf{Z})=0$ and $H_{\mathcal{W}}^{1}(X, j ! \mathbf{Z})=\coprod_{S} \mathbf{Z} / \mathbf{Z}$. We have the exact sequence

$$
0 \rightarrow \operatorname{Ext}(\operatorname{Pic}(V), \mathbf{Z}) \rightarrow H_{\mathcal{W}}^{2}(X, j ! \mathbf{Z}) \rightarrow \operatorname{Hom}(U(V), \mathbf{Z}) \rightarrow 0
$$

and $H_{\mathcal{W}}^{3}(X, j ! \mathbf{Z})=\operatorname{Ext}(U(V), \mathbf{Z})=$ the Pontryagin dual of $k^{*}$.

Proof. We have proven part (a) as part of Proposition 3.4, and part (b) follows from part (a) by Theorem 5.4. For part (c) we claim that $H_{\mathcal{W}}^{i}\left(X, j_{*} \mathbb{G}_{m}\right)$ is naturally isomorphic to $H_{\mathcal{W}}^{i}\left(V, \mathbb{G}_{m}\right)$. Let $\bar{j}$ be the induced map from $\bar{V}$ to $\bar{X}$. We have the two spectral sequences following from Proposition 2.3:

$$
\begin{aligned}
H^{p}\left(\Gamma_{0}, H_{\text {ét }}^{q}\left(\bar{X}, \bar{j}_{*} \mathbb{G}_{m}\right)\right) & \Rightarrow H_{\mathcal{W}}^{p+q}\left(X, j_{*} \mathbb{G}_{m}\right), \\
H^{p}\left(\Gamma_{0}, H_{\text {ét }}^{q}\left(\bar{V}, \mathbb{G}_{m}\right)\right) & \Rightarrow H_{\mathcal{W}}^{p+q}\left(V, \mathbb{G}_{m}\right) .
\end{aligned}
$$

Since $R^{i} \bar{j}_{*} \mathbb{G}_{m}=0$ for $i \geqslant 1$ (see [Mil80, p. 108]), the Leray spectral sequence degenerates, and we conclude that $H_{\text {ét }}^{p}\left(\bar{X}, \bar{j}_{*} \mathbb{G}_{m}\right)=H_{\text {ét }}^{p}\left(\bar{V}, \mathbb{G}_{m}\right)$. Hence the two above spectral sequences agree on their $E_{2}$-terms, and hence in the limit, which proves the claim.

From the exact sequence of sheaves

$$
0 \rightarrow \mathbb{G}_{m} \rightarrow j_{*} \mathbb{G}_{m} \rightarrow i_{*} \mathbf{Z} \rightarrow 0
$$

we get, using the above claim, the exact sequence

$$
\begin{gathered}
0 \rightarrow H_{\mathcal{W}}^{0}\left(X, \mathbb{G}_{m}\right) \rightarrow H_{\mathcal{W}}^{0}\left(V, \mathbb{G}_{m}\right) \rightarrow H_{\mathcal{W}}^{0}(S, \mathbf{Z}) \rightarrow H_{\mathcal{W}}^{1}\left(X, \mathbb{G}_{m}\right) \rightarrow H_{\mathcal{W}}^{1}\left(V, \mathbb{G}_{m}\right) \\
\rightarrow H_{\mathcal{W}}^{1}(S, \mathbf{Z}) \rightarrow H_{\mathcal{W}}^{2}\left(X, \mathbb{G}_{m}\right) \rightarrow H_{\mathcal{W}}^{2}\left(V, \mathbb{G}_{m}\right) \rightarrow 0,
\end{gathered}
$$

which easily yields

$$
0 \rightarrow \operatorname{Pic}(V) \rightarrow H_{\mathcal{W}}^{1}\left(V, \mathbb{G}_{m}\right) \rightarrow \operatorname{Hom}\left(\coprod_{S} \mathbf{Z} / \mathbf{Z}, \mathbf{Z}\right) \rightarrow 0
$$

and $H_{\mathcal{W}}^{2}\left(V, \mathbb{G}_{m}\right)=0$, thus proving part (c).

We next recall from Weibel [Wei94, Exercise 3.6.1] that for $A^{\bullet}$ any complex of abelian groups, we have the exact sequence

$$
0 \rightarrow \operatorname{Ext}\left(h^{1-p}\left(A^{\bullet}\right), \mathbf{Z}\right) \rightarrow h^{p}\left(R \operatorname{Hom}\left(A^{\bullet}, \mathbf{Z}\right)\right) \rightarrow \operatorname{Hom}\left(h^{-p}\left(A^{\bullet}\right), \mathbf{Z}\right) \rightarrow 0 .
$$

Now part (d) follows immediately from part (c), together with the duality theorem (Theorem 5.4) and Lemma 5.3 .

\section{Values of zeta-functions at zero}

In this section we will state the conjecture mentioned in the Introduction and prove it for curves and smooth projective varieties, and a modified form of it for smooth surfaces. Let $k=\mathbf{F}_{q}$ be a finite field. Let $\Gamma$ be the Galois group of $\bar{k}$ over $k$, and let $\phi$ be the Frobenius element of $k$, which sends $x$ to $x^{q}$ and topologically generates $\Gamma$. Let $\Gamma_{0}$ denote the subgroup of $\Gamma$ generated by $\phi$. Let $\theta$ in $H_{\mathcal{W}}^{1}(k, \mathbf{Z})=H^{1}\left(\Gamma_{0}, \mathbf{Z}\right)=\operatorname{Hom}\left(\Gamma_{0}, \mathbf{Z}\right)$ be the homomorphism that sends $\phi$ to 1 .

We also denote by $\theta$ the pullback of $\theta$ to $H_{\mathcal{W}}^{1}(X, \mathbf{Z})$, where $X$ is any scheme over $k$. For any sheaf (or complex of sheaves) $F$ on the Weil-étale site of $X$, there is a natural pairing $F \otimes \mathbf{Z}$ to $F$ induced by $x \otimes n \rightarrow n x$. This pairing induces a map $\bigcup \theta$ from $H_{\mathcal{W}}^{i}(X, F)$ to $H_{\mathcal{W}}^{i+1}(X, F)$. Observe that, since $\theta$ lies in $H^{1}\left(\Gamma_{0}, \mathbf{Z}\right), \theta \cup \theta=0$, so cupping with $\theta$ makes the cohomology groups (or hypercohomology groups) $H_{\mathcal{W}}^{i}(X, F)$ into a complex. Let $h_{\mathcal{W}}^{i}(X, F)$ be the homology groups of this complex.

Definition 7.1. Assume that $h_{\mathcal{W}}^{i}(X, F)$ is finite for all $i$, and zero for $i$ large. Define the Euler characteristic $\chi(X, F)$ to be $\prod_{i} \mid\left(\left.h^{i}(X, F)\right|^{(-1)^{i}}\right.$. 


\section{The WeIL-ÉtAle TOPOLOGY ON SCHEMES OVER FINITE FIELDS}

Assume that we have a cohomological $\delta$-functor $H^{i}$ from an abelian category $\mathcal{A}$ to the category of abelian groups, with $H^{i}=0$ for $i<0$. Assume also that we have a cohomology operation $\theta: H^{i} \rightarrow H^{i+1}$ with square zero for all $i$. Let $A$ be an object in $\mathcal{A}$, and let $h^{i}(A)$ be the homology of the complex $H^{i-1}(A) \rightarrow H^{i}(A) \rightarrow H^{i+1}(A)$, the maps being given by $\theta$. Say that $A$ is ' $\theta$-finite' if $h^{i}(A)$ is finite for all $i$ and $H^{i}(A)$ is zero for $i$ large. In this case, let $\chi(A)=\prod_{i}\left|\left(h^{i}(A)\right)\right|^{(-1)^{i}}$.

Proposition 7.2. (a) If $0 \rightarrow A \rightarrow B \rightarrow C \rightarrow 0$ is exact, and $A, B$ and $C$ are all $\theta$-finite, then $\chi(A) \chi(C)=\chi(B)$.

(b) If $B$ and $C$ are $\theta$-finite, and $H^{i}(B)$ is finite for $i \geqslant 2$, then $A$ is also $\theta$-finite.

Proof. Let $\alpha^{i}$ (respectively $\beta^{i}$ ) (respectively $\gamma^{i}$ ) be the map from $H^{i}(A)$ to $H^{i}(B)$ (respectively $H^{i}(B)$ to $\left.H^{i}(C)\right)$ (respectively $H^{i}(C)$ to $H^{i+1}(A)$ ). Let $D^{i}$ be the image of $\alpha^{i}, E^{i}$ the image of $\beta^{i}$, and $F^{i}$ the image of $\gamma^{i}$. Let $f^{i}$ be $\theta$ on $H^{i}(A), g^{i}$ be $\theta$ on $H^{i}(B)$, and $h^{i}$ be $\theta$ on $H^{i}(C)$.

Let $\delta^{i}$ be the map induced by $\theta$ from $D^{i}$ to $D^{i+1}, \epsilon^{i}$ be the map induced by $\theta$ from $E^{i}$ to $E^{i+1}$, and $\rho^{i}$ the map induced by $\theta$ from $F^{i}$ to $F^{i+1}$. Then we have the commutative diagram:

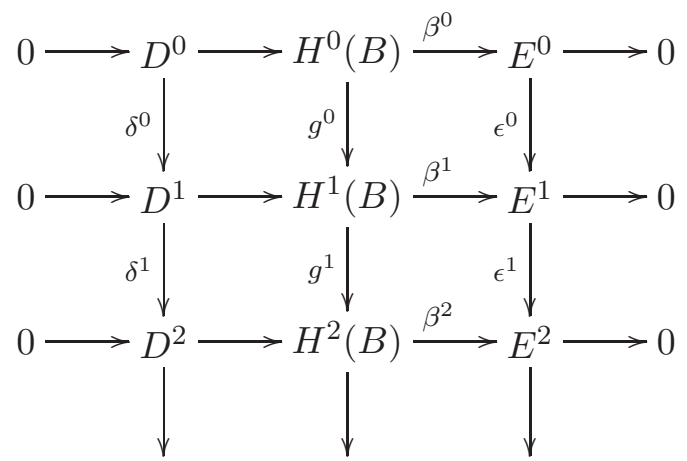

Let $H^{i}(\delta)=\operatorname{Ker}\left(\delta^{i}\right) / \operatorname{Im}\left(\delta^{i-1}\right)$, and similarly for $H^{i}(\epsilon), H^{i}(\beta), H^{i}(f), H^{i}(g)$, and $H^{i}(h)$. It follows from the above commutative diagram that we have an exact sequence

$$
0 \rightarrow H^{0}(\delta) \rightarrow H^{0}(g) \rightarrow H^{0}(\epsilon) \rightarrow H^{1}(\delta) \rightarrow H^{1}(g) \rightarrow H^{1}(\epsilon) \rightarrow \cdots
$$

and similarly we obtain the exact sequences

$$
\begin{aligned}
& 0 \rightarrow H^{0}(\epsilon) \rightarrow H^{0}(h) \rightarrow H^{0}(\rho) \rightarrow H^{1}(\epsilon) \rightarrow H^{1}(h) \rightarrow H^{1}(\rho) \rightarrow \cdots \\
& 0 \rightarrow H^{0}(f) \rightarrow H^{0}(\delta) \rightarrow H^{0}(\rho) \rightarrow H^{1}(f) \rightarrow H^{1}(\delta) \rightarrow H^{1}(\rho) \rightarrow \cdots .
\end{aligned}
$$

We have assumed that $H^{i}(f), H^{i}(g)$, and $H^{i}(h)$ are finite for all $i$. It follows easily by induction that all the groups $H^{i}(\epsilon), H^{i}(\delta)$, and $H^{i}(\rho)$ are also finite.

Since $H^{i}(A), H^{i}(B)$, and $H^{i}(C)$ are zero for $i$ large, so are $D^{i}, E^{i}$ and $F^{i}$. Hence all the Euler characteristics are defined, and with the natural notations, we have

$$
\chi(D) \chi(E)=\chi(B), \quad \chi(A) \chi(F)=\chi(D), \quad \chi(E) \chi(F)=\chi(C),
$$

from which it follows that $\chi(B)=\chi(A) \chi(C)$, and we have proved part (a). A similar and entirely elementary argument proves part (b).

We now state the conjecture.

Conjecture 7.3. Let $U$ be a quasi-projective variety over $k$, and let $j: U \rightarrow X$ be an open dense immersion of $U$ in a projective variety $X$. Assume either that $U$ is smooth or that $U$ is a curve. Then we have the following. 


\section{S. LichtenBAUM}

(a) The cohomology groups $H_{\mathcal{W}}^{i}(X, j ! \mathbf{Z})$ are finitely generated abelian groups, independent of the choice of open immersion $j$, and zero for $i$ large.

(b) The alternating sum of the ranks of the cohomology groups $H_{\mathcal{W}}^{i}\left(X, j_{!} \mathbf{Z}\right)$ is equal to zero.

(c) The order $a_{U}$ of the zero of the zeta-function $Z(U, t)$ at $t=1$ is given by the 'secondary Euler characteristic' $\sum(-1)^{i} i r_{i}$, where $r_{i}=\operatorname{rank}\left(H_{\mathcal{W}}^{i}(X, j ! \mathbf{Z})\right)$.

(d) The homology groups $h_{\mathcal{W}}^{i}(X, j ! \mathbf{Z})$ of the complex $\left(H_{\mathcal{W}}(X, j ! \mathbf{Z}), \theta\right)$ are finite.

Let $Z^{*}(U, 1)$ be $\lim _{t \rightarrow 1} Z(U, t)(1-t)^{-a_{U}}$. Then we have that

(e) $Z^{*}(U, 1)$ is equal, up to sign, to $\chi(X, j ! \mathbf{Z})$.

We remark that part (d) immediately implies part (b).

Theorem 7.4. Conjecture 7.3 is true if $U$ is projective and smooth, or if $U$ is a smooth surface, or if $U$ is a curve. (If $U$ is a smooth surface, we must also take the projective surface $X$ containing $U$ to be smooth.)

Proof. We begin with the case where $U$ is projective and smooth, so $X=U$ and $j$ is the identity.

Lemma 7.5. Let $X$ be projective and smooth. Then Conjecture 7.3 is true for $X$ and the cohomology groups $H_{\mathcal{W}}^{i}(X, \mathbf{Z})$ are finite for $i \geqslant 2$.

Proof. We start with the formula of Milne [Mil86, Theorem 0.1]:

$$
Z^{*}(X, 1)=\left|\left(H_{\text {ét }}^{2}(X, Z)_{\text {cotor }}\right)\right| \prod_{i \geqslant 3}\left|\left(H_{\text {ét }}^{i}(X, \mathbf{Z})\right)^{(-1)^{i}}\right| \operatorname{det}(\delta)^{-1} .
$$

Here $\delta$ is the map induced by cup product with the fundamental class in $H^{1}(\Gamma, \hat{\mathbf{Z}})$ from $H^{0}(X, \hat{\mathbf{Z}})$ to $H^{1}(X, \hat{\mathbf{Z}})$. Note that we have switched here from Milne's use of $\zeta(X, s)$ to $Z(X, t)$, where $t=q^{-s}$, and corrected the obvious misprint $\left(q^{1-s}\right.$ should be $\left.q^{-s}\right)$.

(Milne assumes throughout his paper that $X$ is a variety, i.e. geometrically connected, but his proof of Theorem 0.1 remains valid in the general projective smooth case.) We should point out here that the formula of Milne combines the cohomological description of the zeta-function due to Artin and Grothendieck, a deep theorem of Gabber [Gab83], Deligne's proof of the Riemann hypothesis for varieties over finite fields [Del80], and some sophisticated $p$-adic computations due to Milne. Milne of course also shows that the groups $H_{\text {ét }}^{i}(X, \mathbf{Z})$ are finite for $i \geqslant 3$ and that the cotorsion quotient group of $H_{\text {ét }}^{2}(X, \mathbf{Z})$ is finite. In fact he shows that $H_{\text {ét }}^{2}(X, \mathbf{Z})$ is the direct sum of $\mathbf{Q} / \mathbf{Z}$ and a finite group.

A comparison of the two spectral sequences of Proposition 2.4, part (f), using Lemma 1.1 and the fact that the groups $H_{\text {ét }}^{p}(\bar{X}, \mathbf{Z})$ are torsion for $p>0$, shows that the natural map from $H_{\text {ét }}^{p}(X, \mathbf{Z})$ to $H_{\mathcal{W}}^{p}(X, \mathbf{Z})$ is an isomorphism for $p \geqslant 3$. Since $H_{\text {ét }}^{1}(\bar{X}, \mathbf{Z})=0$, we also have the exact sequence

$$
0 \rightarrow H^{2}\left(\Gamma, H_{\text {ét }}^{0}(\bar{X}, \mathbf{Z})\right) \rightarrow H_{\text {ét }}^{2}(X, \mathbf{Z}) \rightarrow H_{\mathcal{W}}^{2}(X, \mathbf{Z}) \rightarrow 0,
$$

which identifies $H_{\mathcal{W}}^{2}(X, \mathbf{Z})$ with $H_{\text {ét }}^{2}(X, \mathbf{Z})_{\text {cotor }}$, and of course shows that its order is finite.

To show the identity of Milne's Euler characteristic with ours, it only remains to relate our map from $H_{\mathcal{W}}^{0}(X, \mathbf{Z})$ to $H_{\mathcal{W}}^{1}(X, \mathbf{Z})$ given by cup product with $\theta$ to Milne's. But the equality of the orders of the cokernels, which is what is needed, follows immediately from the fact that we have identifications of $H_{\text {ét }}^{i}(X, \hat{\mathbf{Z}})$ with $H_{W}^{i}(X, \mathbf{Z}) \otimes \hat{\mathbf{Z}}$ for $i=0,1$ compatible with the relevant cup products. So we have proved parts (d) and (e) of the theorem, and part (b) is an immediate consequence of part (d). Also, parts (a) and (c) follow immediately from the proof of Theorem 3.1. 


\section{The WeIL-ÉtAle TOPOLOGY ON SCHEMES OVER FINITE FIELDS}

(If $X$ is projective, smooth and geometrically connected, consider the standard representation of the zeta-function $Z(X, t)$ of $X$ as $\prod P_{i}(X, t)^{(-1)^{i+1}}$, with $P_{i}$ the characteristic polynomial of Frobenius. It is immediate that $P_{0}(t)=1-t$, and so has a simple zero at $t=1$. It follows from Deligne's proof of Weil's Riemann hypothesis that the other $P_{i}$ do not vanish at $t=1$, so $Z(X, t)$ has a simple pole there.)

Lemma 7.6. Let $U=X-Z$. Let $j$ be the open immersion of $U$ in $X$ and let $i$ be the closed immersion of $Z$ in $X$. Assume that Conjecture 7.3 holds for $Z$ and that $X$ is projective and smooth. Assume also that the cohomology groups of $U$ with compact support $H_{\mathcal{W}}^{i}(X, j ! \mathbf{Z})$ are finitely generated and independent of the choice of $j$. Then Conjecture 7.3 holds for $U$.

Proof. It follows from Lemma 7.5 that Conjecture 7.3 also holds for $X$ and the cohomology groups $H_{\mathcal{W}}^{i}(X, \mathbf{Z})$ are finite for $i \geqslant 2$. We have the exact sequence of Weil-étale sheaves on $X$ :

$$
0 \rightarrow j ! \mathbf{Z} \rightarrow \mathbf{Z} \rightarrow i_{*} \mathbf{Z} \rightarrow 0 .
$$

It then follows from Proposition 7.2 that $\chi(X, j ! \mathbf{Z})$ is well defined and we have $\chi(X, \mathbf{Z})=$ $\chi(X, j ! \mathbf{Z}) \chi\left(X, i_{*} \mathbf{Z}\right)$. Since we also know that $Z^{*}(X, 1)=Z^{*}(U, 1) Z^{*}(Z, 1)$, we have proven parts (b), (d) and (e) of Conjecture 7.3 and Theorem 7.4 for $U$, and we have assumed part (a) as part of the hypothesis. It remains to prove part (c).

Lemma 7.7. Let $U$ be as in Lemma 7.6. Then we have the following.

(a) The groups $H_{\text {ét }}^{i}\left(\bar{X}, j_{!} \mathbf{Z}\right)$ and $H_{\text {ét }}^{i}\left(\bar{X}, i_{*} \mathbf{Z}\right)$ are semi-simple at zero.

(b) Conjecture 7.3, part (c) is true for $U$.

Proof. Lemma 1.4 immediately implies part (a) of Lemma 7.7.

We have first the exact sequence (modulo torsion)

$$
\begin{gathered}
0 \rightarrow H_{W}^{0}(X, \mathbf{Z}) \rightarrow H_{W}^{0}\left(X, i_{*} \mathbf{Z}\right) \rightarrow H_{W}^{1}(X, j ! \mathbf{Z}) \rightarrow H_{W}^{1}(X, \mathbf{Z}) \\
\rightarrow H_{W}^{1}\left(X, i_{*} \mathbf{Z}\right) \rightarrow H_{W}^{2}(X, j ! \mathbf{Z}) \rightarrow 0 .
\end{gathered}
$$

By comparing this sequence with the exact sequence (modulo torsion)

$$
0 \rightarrow H_{\text {ét }}^{0}(\bar{X}, \mathbf{Z}) \rightarrow H_{\text {ét }}^{0}\left(\bar{X}, i_{*} \mathbf{Z}\right) \rightarrow H_{\text {ét }}^{1}(\bar{X}, j ! \mathbf{Z}) \rightarrow 0
$$

and using the semi-simplicity, we see that the rank of $H_{W}^{1}(X, j ! \mathbf{Z})$ is equal to the rank of $H_{W}^{0}\left(X, i_{*} \mathbf{Z}\right)$ -1 , and the rank of $H_{W}^{2}(X, j ! \mathbf{Z})$ is equal to the rank of $H^{1}\left(X, i_{*} \mathbf{Z}\right)-1$. Also the rank of $H_{W}^{i}\left(X, j_{!} \mathbf{Z}\right)$ is equal to the rank of $H^{i-1}\left(X, i_{*} \mathbf{Z}\right)$ for $i \geqslant 3$.

It then follows, assuming Conjecture 7.3 for $Z$, that the 'secondary Euler characteristic' of $U$ is equal to the difference of the 'secondary Euler characteristic' of $X$ and the 'secondary Euler characteristic' of $Z$. Since the same relationship holds for the orders of the respective zeros of the zeta-functions at $t=1$, we have proved part (b) of Lemma 7.7.

Hence we have now also proved Lemma 7.6.

Now we return to the proof of the original theorem. Lemmas 7.5 and 7.6 immediately yield Theorem 7.4 for smooth curves. Then an argument essentially identical to that used in the proof of Lemma 7.6 yields the result for all curves, by comparing an arbitrary curve to a smooth open dense subset. Finally the result for any smooth surface $U$ follows immediately by using Lemma 3.2 (applied to the projective curve $X-U$ ), Lemma 7.5 and Lemma 7.6. 


\section{The WeIL-ÉtAle TOPOLOGY ON SCHEMES OVER FINITE FIELDS}

\section{REFERENCES}

Del80 P. Deligne, La conjecture de Weil II, Publ. Math. Inst. Hautes Études Sci. 52 (1980), 137-252.

Den84 C. Deninger, On Artin-Verdier duality for function fields, Math. Z. 188 (1984), 91-100.

Gab83 O. Gabber, Sur la torsion dans la cohomologie l-adique d'une variété, C. R. Acad. Sci. Paris Sér. I Math. 297 (1983), 179-182.

Mil80 J. S. Milne, Étale cohomology (Princeton University Press, Princeton, NJ, 1980).

Mil86 J. S. Milne, Values of zeta functions of varieties over finite fields, Amer. J. Math. 108 (1986), 292-360.

Ser59 J.-P. Serre, Groupes algèbriques et corps de classes (Hermann, Paris, 1959).

Wei94 C. A. Weibel, An introduction to homological algebra, Cambridge Studies in Advanced Mathematics, vol. 38 (Cambridge University Press, Cambridge, 1994).

S. Lichtenbaum Stephen_Lichtenbaum@brown.edu

Mathematics Department, Brown University, 151 Thayer Street, Providence, RI 02912, USA 